\title{
Video Recording
}

National Cancer Institute

\section{Source}

National Cancer Institute. Video Recording. NCI Thesaurus. Code C91063.

The storing or preserving of video images to be displayed later. 